\title{
Extended-range fiber polarimetric strain sensor
}

\author{
R. M. Taylor, D. J. Webb, J. D. C. Jones, and D. A. Jackson \\ Physics Laboratory, The University, Canterbury, Kent CT2 7NR, UK
}

Received March 31, 1987; accepted June 25, 1987

\begin{abstract}
We describe a frequency-modulation technique that is applicable to two-beam interferometric systems illuminated by semiconductor diode lasers. The technique permits a determination of the optical path difference between the two arms of the interferometer and is used here to extend the range of a fiber polarimetric strain sensor by determining the order of the particular polarimetric fringe under consideration.
\end{abstract}

In recent years, the development of optical sensors using monomode-fiber sensing elements has become an area of active research. The transduction mechanism is the phase modulation of the guided beam by the applied measurand. A range of signal-processing techniques has been reported that may be classified into three groups, each of which is appropriate for different ranges of measurement resolution. The first group comprises devices that are true interferometers and employ direct phase demodulation ${ }^{1}$; in the second group the differential phase retardance between polarization eigenmodes in a birefringent sensing element is recovered ${ }^{1}$; and in the third, the optical source is frequency modulated and the path-length imbalance in the interferometer is determined from the harmonic content of the output signal. ${ }^{2}$

Interferometric techniques offer the highest resolution, but because of the periodic nature of their transfer function the available unambiguous measurement range is limited. This difficulty may be alleviated through the use of long-range phase-tracking techniques, but even these will fail when the system is reset to its power-up condition, at which point the order of interference cannot be identified. Polarimetric techniques are intrinsically less sensitive and hence offer an increased measurement range at the cost of concomitantly reduced resolution. Polarimetric and interferometric techniques may be combined to yield the wide measurement range of the polarimeter with the resolution of the interferometer. ${ }^{3}$ Frequencymodulated continuous-wave (FMCW) techniques offer the widest measurement range but have significantly poorer resolution than either polarimetric or interferometric techniques.

We describe here a processing scheme for the monomode-fiber sensor in which FMCW and polarimetric techniques are applied simultaneously in order to achieve the widest possible measurement range with enhanced resolution. The system has been demonstrated in the measurement of strain, although it is equally applicable to a range of other measurands.

The propagation of a beam through a linearly birefringent optical fiber is described by ${ }^{4}$

$$
\mathbf{E}_{s}=F \mathbf{E}_{0 s} ; \quad F=\left[\begin{array}{cc}
\exp \left(i \phi_{f}\right) & 0 \\
0 & \exp \left(i \phi_{s}\right)
\end{array}\right],
$$

where $\mathbf{E}_{s}$ and $\mathbf{E}_{0 s}$ are the electric field vectors of the incident and emergent beams, respectively, and $F$ is a pseudo Jones matrix describing the fiber; $\phi_{f}$ and $\phi_{s}$ are the phase retardances for the fast- and slow-polarization eigenmodes, respectively. These phase retardances are functions of axial strain applied to the fiber such that

$$
\frac{\delta \phi_{j}}{\delta l}=\frac{2 \pi}{\lambda}\left(n_{j}+l \frac{\delta n_{j}}{\delta l}\right) ; \quad j=f, s,
$$

where $n_{j}$ is the effective core refractive index of the relevant eigenmode, $l$ is the length of the fiber, and $\lambda$ is the vacuum wavelength of the source. ${ }^{3}$ Either of the $\phi_{j}$ may be determined by using an interferometer. For example, the fiber may form one arm of a MachZehnder interferometer containing a polarization analyzer that selects only one of the eigenmodes. The output intensity is then given by

$$
I_{I}=I_{0 I}\left[1+k_{I} \cos \left(\phi_{j}-\theta_{I}\right)\right],
$$

where $I_{0 I}$ is an intensity constant, $k_{I}$ is a visibility constant, and $\theta_{I}$ is a constant phase term associated with the reference arm of the interferometer. Similarly, the phase difference between the eigenmodes may be determined without an interferometer by placing a polarization analyzer in the output beam at $45^{\circ}$ to the eigenaxes, giving

$$
I_{P}=I_{0 P}\left[1+k_{P} \cos \left(\phi_{f}-\phi_{s}-\theta_{P}\right)\right],
$$

where $I_{O P}, k_{P}$, and $\theta_{P}$ are constants.

The objective of any signal-processing scheme is to recover the phase $\phi_{j}$. A range of techniques exists for determining this quantity within modulo $2 \pi$, but an absolute determination is more difficult. In the FMCW technique, the optical frequency of the source is modulated, thus producing phase modulation in an unbalanced interferometer. The problem is effectively that of determining the net effective optical pathlength imbalance in the interferometer, $L$, where

$$
\phi_{j}-\theta_{I}=\frac{2 \pi L}{\lambda} \text {. }
$$

Hence, for an optical source of nominal frequency $\nu$ modulated by an amplitude $\Delta \nu$ with circular modulation frequency $\omega$, 


$$
\phi_{j}-\theta_{I}=\frac{2 \pi L}{c}(\nu+\Delta \nu \sin \omega t) \text {. }
$$

The sinusoidal modulation of optical frequency is readily achieved by using a diode-laser source, where optical frequency is dependent on injection current. ${ }^{5}$ The frequency spectrum of the interferometer may be found by combining Eqs. (3) and (6) and expanding in terms of Bessel functions to give

$$
\begin{aligned}
I_{I}= & I_{0 I}\left\{1+k_{\mathrm{I}} \cos \chi\left[J_{0}(\Delta \chi)+2 \sum_{l=1}^{\infty} J_{2 l}(\Delta \chi) \cos 2 l \omega t\right]\right. \\
& \left.-k_{I} \sin \chi\left[2 \sum_{l=1}^{\infty} J_{2 l-1}(\Delta \chi) \sin (2 l-1) \omega t\right]\right\}
\end{aligned}
$$

where $\chi=2 \pi L \nu / c$ and $\Delta \chi=2 \pi L \Delta \nu / c$. This spectrum is simplified if the interferometer is at quadrature; that is, for the condition $\chi=\pi / 2+2 N \pi$, where $N$ is an integral. Under these conditions, the spectrum of Eq. (7) contains only odd harmonics. In particular, the amplitude of the signal at the frequency of the modulation is proportional to $J_{1}(\Delta \chi)$, which is in turn proportional to $L$ in the small-signal limit. Hence, in principle, a measurement of the output signal at the frequency of modulation would enable the optical path difference to be determined. The technique also permits accurate balancing of the interferometer. ${ }^{6}$

Figure 1 shows the experimental arrangement used to investigate the variation in the signal close to zero path-length imbalance. A Mach-Zehnder configuration was used, incorporating a piece of highly birefringent fiber in one arm. Circularly polarized light was launched into this fiber to populate both eigenmodes equally. Interferometric fringes were obtained by interfering light in one of the eigenmodes with that from the reference arm. Complementary outputs were obtained at detectors D1 and D2 and used in conjunction

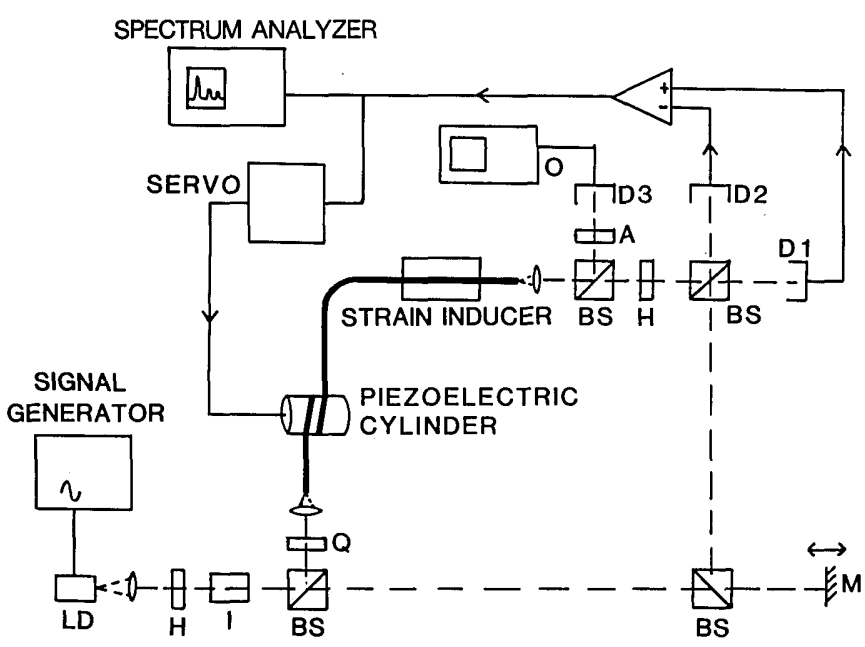

Fig. 1. Experimental configuration. LD, laser diode; H's, half-wave plates; $\mathrm{Q}$, quarter-wave plate; I, isolator; BS's, beam splitters; A, analyzer; O, oscilloscope; M, mirror; D1D3, photodetectors. Dashed lines, optical beam; thick lines, monomode fiber; arrows, electronic signal.

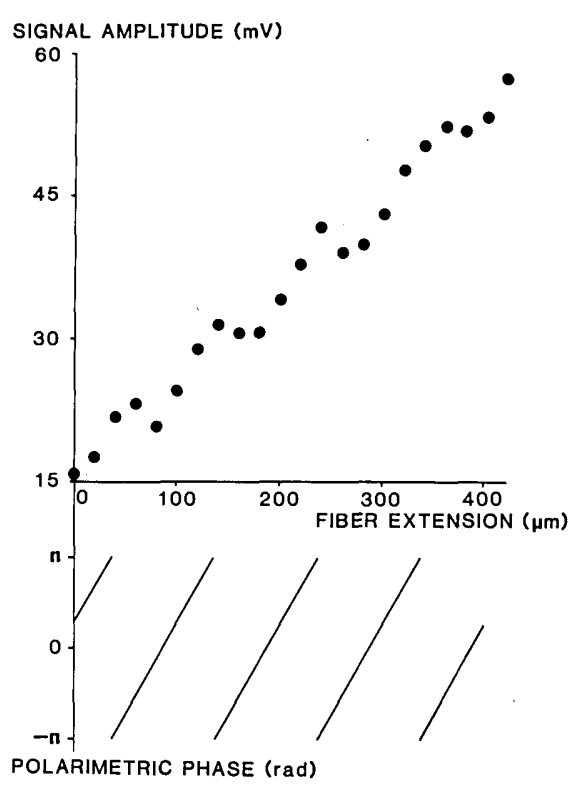

Fig. 2. Experimental results. Upper trace, amplitude of signal at modulation frequency, from D1 and D2; lower trace, polarimetric output, from D3 (see text).

with low-gain bandwidth product servoelectronics and a fiber-wound piezoelectric cylinder to hold the interferometer in quadrature. ${ }^{7}$ Mirror $M$ was adjusted so that this interferometer was within a few hundred micrometers of zero-path imbalance. Polarimetric fringes were observed at detector D3. A strain was introduced in approximately $15 \mathrm{~cm}$ of the fiber by using a microtranslator. The locking range of the servo system was in the region of a few micrometers, and the servo was reset every time the microtranslator was adjusted. The laser diode used was a Sharp Model LT010 emitting at approximately $816 \mathrm{~nm}$. The amplitude of the sinusoidal frequency modulation was approximately $4 \mathrm{GHz}$, and the modulation frequency was $1 \mathrm{kHz}$.

Figure 2 shows the results obtained in the experiment. The upper trace shows the amplitude of the signal at the modulation frequency, $J_{1}(\Delta \chi)$. An approximately linear relationship is observed between this amplitude and the applied strain. The small periodic departure from linearity observed corresponds to interference between the eigenmodes of the birefringent fiber used and arises because of the imperfect polarizing components used. The lower trace shows the polarimetric transfer function obtained from detector D3.

The FMCW information (upper trace) is used only to identify the order of the polarimetric interference (lower trace), and hence the imperfect response observed does not degrade the result obtained. A range of techniques exists for the accurate determination of the polarimetric phase (see, for example, Ref. 1 ). It is hence feasible to determine the applied strain with the high resolution of the polarimeter but with the wide measurement range of the FMCW technique. For example, in the present experiments a maximum strain of $420 \mu \mathrm{m}$ was applied. Although the phase resolution of the polarimeter was not measured in this 
experiment, typical values lie in the range $1 \mu \mathrm{rad}$ to 1 mrad. ${ }^{1}$ The polarimetric sensitivity coefficient was $2 \pi \times 10^{4} \mathrm{rad} \mathrm{m}^{-1}$, so that with a phase resolution of 1 mrad a strain resolution of about $10 \mathrm{~nm}$ would be obtained.

The resolution of the FMCW technique is fundamentally limited by shot noise in the photodetector current and may be calculated by setting the change in photodetector current due to a small change in optical path equal to the shot noise due to the mean photodetector current. If the minimum detectable change in optical path is $\Delta L$, then

$$
\frac{2 \pi \alpha k_{I} \Delta L \Delta \nu I_{0 I}}{c}=\left(2 e \alpha I_{0 I} B\right)^{1 / 2},
$$

where $\alpha$ is the responsivity of the photodetector, $B$ is the bandwidth of the system, and $e$ is the electronic charge. Taking typical values of $\alpha, k_{I}, \Delta \nu$, and $I_{0 I}$ as $0.5,1,10 \mathrm{GHz}$ and $100 \mu \mathrm{W}$, respectively, and a $10-\mathrm{Hz}$ bandwidth suggests a shot-noise-limited resolution of around $1 \mathrm{~nm}$. In practice this limit is not approached because of additional noise factors arising from the source and the environment. The laser source exhibits both intensity and phase noise. The effects of intensity noise may be largely eliminated by using complementary interferometric outputs, as we have done. The effects of phase noise on the output of the interferometer are proportional to the optical path difference between the signal and reference arms. ${ }^{8}$ Since our technique operates near the point of zero optical path difference, the limit on the resolution of a practical system is likely either to be environmental in nature (acoustic or temperature effects) or to arise from the nonideal nature of the components used, as we observed.
An important enhancement in this technique would be achieved if the resolution of the FMCW technique were sufficient to obtain the order of interference in the interferometric output. In our experiments we observed a ratio in sensitivity between the interferometric and polarimetric responses of 1:112. Even without this refinement, the interferometric information may still be derived since the order of interference may be found from a combination of the FMCW and polarimetric information. However, the additional complexity in the signal processing required would not generally be warranted.

Finally, with the current interest in polarizationmaintaining fiber couplers and other fiber components, an all-fiber version of this system should be possible in the near future, which would considerably enhance its usefulness.

This research was partly supported by GEC Avionics and Kent Scientific and Industrial Projects Ltd.

\section{References}

1. D. A. Jackson, J. Phys. E 18, 981 (1985).

2. D. Uttam and B. Culshaw, IEEE J. Lightwave Technol. LT-3, 971 (1985).

3. J. D. C. Jones, P. Akhavan Leilabady, and D. A. Jackson, Int. J. Opt. Sensors 1, 123 (1986).

4. A. J. Barlow, IEEE J. Lightwave Technol. LT-3, 135 (1985).

5. D. Welford and S. Alexander, IEEE J. Lightwave Technol. LT-3, 1092 (1985).

6. A. Dandridge, IEEE J. Lightwave Technol. LT-1, 514 (1983).

7. D. A. Jackson, R. Priest, A. Dandridge, and A. B. Tveten, Appl. Opt. 19, 2926 (1980).

8. A. Dandridge and A. B. Tveten, Appl. Phys. Lett. 39, 530 (1981). 